\title{
AC 2012-4539: THE COMPLEXITIES OF ENGINEERING DESIGN AND SYSTEM MODELING
}

\section{Dr. Gayle E. Ermer, Calvin College}

Gayle Ermer is a professor of engineering at Calvin College in Grand Rapids, Mich. She teaches in the mechanical concentration in the areas of machine dynamics and manufacturing processes. Her master's degree was obtained from the University of Wisconsin, Madison, in manufacturing systems engineering (1987), and her Ph.D. from Michigan State University (1994). Her research interests include philosophy of technology, engineering ethics, and women in engineering. 


\title{
The Complexities of Engineering Design and System Modeling
}

\begin{abstract}
One of the many challenges facing the engineering profession and its system of engineering education is the need for effective problem solving and decision making in the midst of the increasing complexity of contemporary technological systems. Simple observation reveals that engineered products are becoming more complicated over time. Perhaps more importantly, the interactions between technological artifacts and the humans and societies who create and use them, as well as the interactions between the technological artifacts and the living world in which they are embedded, are multiplying. Understanding the nature of these interactions is crucial to the effectiveness of system operation and the reduction of risk.
\end{abstract}

Engineers are currently taught to deal with this complexity by relying on predictions of system behavior based on the abstract reductionist models of science. These models are typically deterministic and rely on modernist approaches to understanding reality. This paper will explore and categorize some of these engineering modeling approaches. Also, in order to encourage the development of a more comprehensive approach to modeling which includes the contexts in which physical systems are implemented, some engineering educators have tried to augment reductive models with consideration of a comprehensive set of design norms. This paper will present a set of norms that point to the need for a more expansive toolbox of modeling approaches to better understand system behavior at multiple levels. These norms address different aspects of the structure of lived reality and therefore attempt to take into account the complexity and the situated-ness of engineered solutions.

Finally, the relatively recent development of the inter-disciplinary field of complexity theory suggests some new strategies for approaching engineering system design. Complexity theory attempts to describe and model certain classes of systems, particularly biological systems, which exhibit characteristics such as rich interaction between components, non-equilibrium responses, and emergent behavior. This paper will describe some conceptual modeling approaches to dealing with complexity for the purpose of identifying new tools or principles for improving engineering design. Finally, some recommendations will be made as to how to integrate the most useful insights of complexity theory into the engineering curriculum.

\section{Introduction}

It seems obvious that today's engineered systems are more complex than those of the past. Simple observation reveals that the number of individual components contained in many products has increased over time. Perhaps more importantly, the interactions between these technological artifacts and the humans and societies who create and use them, as well as the interactions between the technological artifacts and the living world in which they are embedded, are multiplying. A recent article from Mechanical Engineering magazine personalizes this challenge: "Engineers don't have the luxury of ignorance. We who design these complex systems have to understand how the various components of a system fit together and anticipate how the interactions between these components could lead to failure."i 
The recent failure of the BP Deepwater Horizon oil well in the Gulf of Mexico is a good example of this phenomenon. ${ }^{\text {ii }}$ The need to find more sources of oil has led to the prospect of drilling at ever greater ocean depths. This exposes oil rigs to harsher conditions in more isolated environments than previous rigs. It also exposes a diverse geography of plant and animal life to consequences of a failure. The business models underlying the oil industry encourage specialization of the different technological aspects of running a rig, with entirely different organizations involved in the design, build, and operation phases. In the design process, engineers need to anticipate all of these interactions between the many individuals and companies. There are many complexities in the path from mechanical design to successful implementation of a system in real-world conditions.

So, the challenge facing the engineering profession and its system of engineering education is the need for effective problem solving and decision making in complex situations. Different manifestations of complexity have to be addressed in the context of engineering design in order to produce products and infrastructure that are safe and effective. This paper will explore various aspects of complexity in technology, particularly in engineering design and analysis, and suggest some ways that engineering education may need to be adjusted to address these needs, with a special focus on the complexities addressed by the interdisciplinary field of complexity theory.

\section{Complexity in Technology}

For some, it may be intuitively obvious that technology is increasing in complexity, based on the increasing scale and scope of our technological projects. However, our perception of the extent to which this is occurring is likely influenced by our own interpretation of the term "complexity." While it is not my intention to do a detailed etymological analysis of this word, I would like to highlight some aspects of its meaning that impact engineering design.

\section{Complex $=$ Complicated}

Dictionary definitions (as well as every day usages) of the word "complex" primarily emphasize a sense of complexity as complicatedness. According to the Oxford dictionary, the adjective form of "complex" means "consisting of many different and connected parts." iii When engineers use the term complex, they are often referring to an engineered system that has many components or parts, integrates many process steps, and relates to multiple stakeholders. It may also refer to a large number of alternatives for the many components or process steps as the engineering design process is carried out. A search of ASEE conference papers published since 1997 containing "complex" or "complexity" in the title yields 55 entries. By my appraisal, 46 primarily used the term in this sense. In many of these cases the term is taken to mean the opposite of simple and to be synonymous with large-scale. Increasing complicatedness is clearly a challenge to designers, because of the difficulty of integrating all of the different components correctly into models for predicting system behavior and the difficulty of managing a large collection of inputs. More complicated systems increase the risk of errors in the models going undetected. Computational software is of considerable help in the face of this type of complexity. Many of the papers referred to above present techniques for solving large sets of equations or otherwise improving the analysis process for complicated systems. ${ }^{\text {iv }}$ 
W. Bryan Arthur, in his book The Nature of Technology, describes the inner structure of technological innovation in a way that explains the phenomenon of increasing complicatedness over time. His thesis is that technology evolves, i.e. it is descended from previous technologies by re-combination and appropriation of new phenomena. He calls this process "combinatorial evolution", (as opposed to the natural selection based on random mutation mechanism of biological evolution). If, as he postulates, new technologies come into being as fresh combinations of what already exists, then it stands to reason that as the number of currently existing building blocks increases, the number of possible combinations will also be vastly increased. Existing technologies provide "a vocabulary of elements that can be put together programmed - in endlessly novel ways for endlessly novel purposes."vi Proliferation of elements means that over time many more of them need to be described, analyzed and integrated as part of the design process.

\section{Complex $=$ Difficult to Comprehend}

A second sense of the nature of complexity can also be found in the Oxford dictionary, where the word complex is defined as "not easy to analyse or understand.",vii In engineering work, the term complex is used in naming systems or concepts that are abstract and therefore hard to visualize. It is also applied to problems that are nuanced or ambiguous, whose solutions are elusive. ${ }^{\text {vii }}$ Complicatedness and incomprehensibility often go hand in hand. Having large numbers of components and interactions in a system often makes the behavior of the system harder to visualize and anticipate. Much engineering education research is devoted to developing techniques for making these sorts of complex concepts accessible to students. ${ }^{\text {ix }}$ This type of complexity also captures the sense that our models are challenged to represent the real behavior of systems because of required assumptions and simplifications. Some aspects of the system are difficult to translate into mathematical models. Sometimes the difficulty in making design decisions is influenced by the complexity of addressing components or aspects of the design that are not commensurate because they traditionally belong to different domains of analysis (e.g. integrating voltages with mechanical stresses or costs with reliability data).

\section{Complex $=$ Non-Deterministic}

For many engineering problems in the previous two categories, it may be difficult to find a solution, but in principle optimization and determination of the best course of action is possible. On the other hand, many real life engineering problems have solutions that cannot be directly obtained from the information available as inputs to the problem. This is because nearly all engineering designs are complex in the sense that they are open-ended. Complexity is introduced because of the fact that the problem is under-constrained. Multiple solutions might work and it is not immediately clear which is the best. Therefore solutions cannot be arrived at purely by deductive logic, but may be historically contingent or based on individual or organizational preferences. Another contribution to the complexity of indeterminacy is that systems are subject to random effects and unpredictable variations which must be compensated for in the design in order to avoid unanticipated failures.

Complex $=$ Socially Enmeshed 
The final aspect of complexity in engineered systems that I would like to identify is the entwinement of technological and social values that impacts engineering work. It is a challenge to anticipate what requirements should be placed on new designs due to societal needs (e.g. how safe does a nuclear plant have to be to be considered safe?). It is also difficult to understand how a given technological system will influence the culture in which it is embedded.

Thomas P. Hughes emphasizes this aspect of complexity in his technological momentum theory of history. In his view, a new technological development, although generated by societal needs, will have a life of its own and tend to resist control or direction by society. An example he uses to illustrate this view is the development of synthetic gasoline from coal by hydrogenation in post-World War I Germany.

"The history of hydrogenation in Germany between 1914 and 1933 reveals, in summary, a technology stimulated by war gathering a momentum carrying over into peacetime. The commitment of engineers, chemists, and managers experienced in the process, and of the corporation heavily invested in it, contributed to this momentum. The product of the wartime hydrogenation process was applied to peaceful purposes, but this did not entirely absorb the creativity of the engineers and chemists looking for new applications of the challenging technology they had mastered."x

Although hydrogenation was originally developed as a technique for providing nitrogen for fertilization of food crops, it eventually was used to produce synthetic fuel that contributed to the success of Hitler's offensive in World War II. Technological systems impact society and society impacts technological systems in ways that are difficult to understand and predict. This contributes to the "messiness" or complexity of technological problems.

Townsend and Neeley illustrate the complexity of social interactions in the history of technology using the example of the introduction of sewing machines in the $19^{\text {th }}$ century. The same sewing machine that "eases the burden of daily living"xi for middle class women also contributed to the enslavement of women and children in industrial sweatshops. In the words of the poet Adrienne Rich, historians of technology often "fall short of "naming the complexity' involved in the interaction between technology and the social fabric." xii

All of these aspects of complexity (complicatedness, incomprehensibility, in-determinacy and social enmeshment) have in common the acknowledgement of interactions. These interactions are present between and among system sub-components, and also between elements of the system and its environment. Engineering design needs to reflect an accurate and robust understanding of these interactions in order to successfully predict overall system behavior. The next section of this paper will present techniques currently employed to address these complexities in the traditional engineering curriculum.

\section{Engineering Modeling}

The central activity of the engineering profession involves creative design of new systems in response to given needs. Design is a process of problem-solving, consisting of many stages between the development of a new concept and the implementation of a concrete solution. It is 
about bringing ideas to life. A structured engineering design process is commonly taught and used to help clarify and reduce the complexity of engineering design work. A typical prescriptive flow chart for this process, taken from a popular first-year engineering design textbook, is shown in Figure 1. ${ }^{\text {xiii }}$

The modeling/analysis/evaluation stage of the design process is the focus of this paper, particularly because this is what is emphasized in traditional engineering undergraduate education. The success of a designed system depends on many things, but especially on careful analysis and evaluation that takes into account all significant aspects of a system's behavior. Inadequate or imprecise analysis can contribute to ineffectiveness of the system or to potential failure, perhaps even disaster. According to Bob Colwell in an editorial for Computer, "The real art of engineering, its sine qua non, is in evaluating a proposed design from every angle and vantage point to make sure a design will achieve its goals and prove reliable over its intended lifespan." "xiv As part of analysis, engineers construct models for basic understanding, prediction and control. George Box stated, as part of his work in statistics and experimentation, that: "All models are wrong, but some are useful." ${ }^{\mathrm{xv}}$ I would suggest that models are not just useful, but necessary to responsible engineering design.

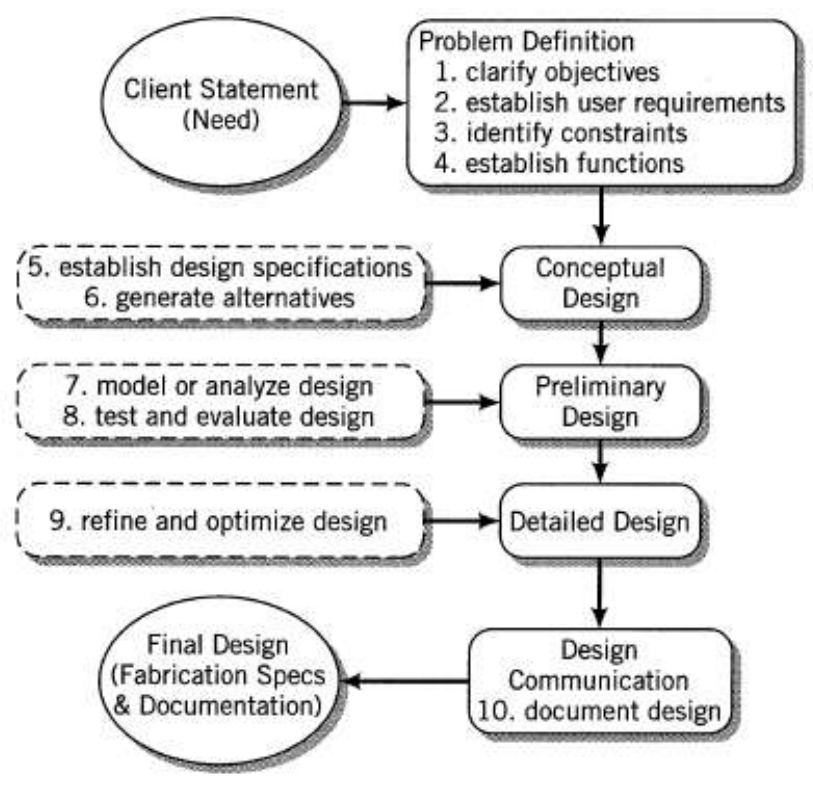

Figure 1. Design Process (Dym and Little)

\section{Traditional Modeling Techniques}

Traditionally, scientific reductionism has been the basic approach to engineering system behavior modeling. Even the structured design process itself is an attempt to reduce a spontaneous, iterative, and open-ended activity into discrete, sequential steps. Reductionism is useful because it helps develop general understanding by isolating or abstracting certain aspects from the holistic reality of the system in order to identify patterns, establish cause and effect, and 
identify mathematical relationships between variables. In order to predict system behavior (and therefore to make choices about how to appropriately design a given system), the system is subdivided into chunks that can be understood and mathematically modeled, or simulated, in order to predict system behavior.

Of reductionist models, the most prevalent in the engineering curriculum are deterministic, expressed in equations derived from scientific theory. Equations are developed such that a given set of variable values generates a single behavior prediction. For example, the output angular position of a four bar linkage can be determined from the solution of a quadratic equation. Or, a free body diagram is used to write force balance equations which can be used to solve for unknown forces in the linkage. These types of models are the primary focus of first and second year engineering courses like statics and dynamics, and are used and extended in upper level courses as well. Students can come away from learening deterministic models with the idea that there is always a single correct answer to every problem. These models demonstrate significant explanatory power, but are only practical for relatively small systems with simple interactions, based on assumptions which might not apply in the real world. The effect of multiple courses which teach primarily deterministic models might be an over-estimation by students of their applicability in engineering design. Of course, not all deterministic models are theoretical. There has always been a recognition among engineers that experimentation is needed to verify design behavior, but often, since it is not practical to test a prototype early in the design process, experiments based on simplified models are used (for example, material property data obtained from a standard tensile test).

To allow engineering students and practicing engineers to better predict the behavior of systems that are too complicated to be handled with explicit equation solutions, numerical modeling techniques have been developed. Numerical solutions allow engineers to simulate the behavior of systems that are too complicated to be modeled with straight-forward explicitly derived equations. The most common numerical modeling approach in mechanical engineering is finite element analysis (FEA). A complicated geometry can be subdivided into many elements of simpler geometry whose behavior and interactions are better understood and predicted. A digital computer can then be used to solve simultaneously the many equations used to represent the system connections and externally applied constraints. The danger of these models is that they tend to promote a black box approach to behavior prediction. Students can enter data, let a computer churn out a solution, and may unquestionably accept the results. It is difficult to determine if an answer obtained in this way is reasonable unless a parallel modeling method is available or a significant level of experience with the systems being modeled has been obtained. The more complicated the system, the more difficult it becomes to identify a possible error in the model.

The recognition that some system variables exhibit unpredictable or random variation in values has led to the incorporation of statistical modeling into some areas of the curriculum. Stochastic models can aid in predicting system behavior in situations where a specific state may not be known, but average behaviors based on assumptions about variability can help to understand and predict future states. In mechanical engineering, statistical process control that can be used to analyze and control the behavior of manufacturing systems is taught, along with basic estimation of error in measurements. For systems that do not lend themselves to detailed analysis, engineers 
have also relied on simple heuristic models based on past experience to make better design decisions. Typically, the total amount of content related to stochastic modeling and heuristics in the curriculum is still relatively small compared deterministic modeling content.

Engineering education is about building a toolbox for students that will enable them to contribute to successful engineering design analysis in the future. As engineering educators, we need to ensure that this repertoire of knowledge and skills contains a robust variety of modeling tools that can be used for all of the different systems that might be designed. The modeling tools previously described address primarily the complicatedness aspect of complexity.

\section{Normative Design and Complexity}

In order to address higher level complexities in engineering system analysis (particularly indeterminacy and social enmeshment), a multi-aspectual normativity framework for technology design can be used. This framework is described in the book Responsible Technology. This approach identifies a number of aspects that can be abstracted from the complex nature of reality and need to be considered as part of the engineering design process. ${ }^{\text {vi }}$ Each of these aspects involves normative judgments that are appropriate to the particular aspect identified. A complete list of aspectual norms, along with brief descriptions, is included in Table $1 .{ }^{\text {xii }}$ The norms are typically applied in determining a comprehensive set of design specifications for a system (items 1-5 of Figure 1). I believe that this normative framework can also be helpful in guiding the modeling and analysis aspects of engineering design (items 6-9 of Figure 1), at least in stimulating questions about new modeling techniques that may need to be incorporated to address the interactions between engineered objects and individuals and societies who operate them.

Normative design principles are derived from aspects of the structure of reality that cannot be reduced to or predicted by structures from a lower level. For example, the economic aspect of a design identified in the stewardship norm involves variables related to historical development (cultural appropriateness) and personal communication (transparency). But, the economic aspects of behavior are not determined completely by historical and language-related variables. The behavior of economic systems can be seen as "emerging" from historical and communicational components, but analysis of economic behavior involves application of a set of principles appropriate to the economic domain.

Kalsbeek provides an example of the launching of a manned space vehicle to illustrate the aspects. ${ }^{\text {viii }}$ The economic aspect (stewardship norm) will involve calculations of the costs involved. The social and lingual aspects (addressed in the transparency norm) will involve the relationships between engineers and crew members and the language and symbols used for communication. The historical aspect (cultural appropriateness) will involve determination of whether the methods and technology available are capable of ensuring accomplishment of the launch. Each of these aspects needs to be modeled (along with the physical and biotic aspects typically addressed by scientific models) in order to make the launch a success.

The implicit lessons of using a normative aspectual framework for design are 1) that physical laws/models are necessary for analyzing physical problems, 2) that biological laws/models are 
necessary for analyzing biological problems, 3) that psychological laws/models are necessary for analyzing psychological problems, etc. All of the aspects that contribute to a particular engineered system must be accounted for by models of appropriate level to ensure a complete evaluation of the system in its context. The norms may also highlight areas of the system where tradeoffs might need to be made. Complex engineering problems imply that system boundaries must be extended to include all relevant interactions and that system models be included that are appropriate to all the levels of system behavior. The norms remind us that the system needs to be defined so that models of the system will be as complex as they need to be to cover all aspects of system behavior. The norms provide a framework for integrating the participation of human actors and societal implications into engineering analysis. This may point us toward integrating modeling types from other disciplines into our design evaluation.

Table 1. Design Norms (Responsible Technology)

\begin{tabular}{|l|l|}
\hline $\begin{array}{l}\text { Cultural } \\
\text { Appropriateness }\end{array}$ & Technology should fit the society in which it will be implemented \\
\hline Transparency & $\begin{array}{l}\text { The composition and use of technology should be clear to all, } \\
\text { including non-technologists, and the risks and benefits should be } \\
\text { honestly communicated }\end{array}$ \\
\hline Stewardship & $\begin{array}{l}\text { All resources should be used frugally and efficiently, and the effects of } \\
\text { technology on the natural environment minimized }\end{array}$ \\
\hline Harmony & $\begin{array}{l}\text { The design should be pleasing to use, attractive, and promote healthy } \\
\text { relationships }\end{array}$ \\
\hline Justice & Benefits and burdens of technology should be allocated fairly \\
\hline Caring & $\begin{array}{l}\text { Technological solutions should address real needs and express care for } \\
\text { all individuals }\end{array}$ \\
\hline Trust & $\begin{array}{l}\text { Technology should be reliable and worthy of trust on the part of those } \\
\text { who make and use it }\end{array}$ \\
\hline
\end{tabular}

\section{Truly Complex Systems and Complexity Theory}

Up until now, this paper has been using broad, commonly understood interpretations of the term complexity. This section of the paper will use the term complexity in a much more narrow sense, referring to systems that display particular types of behavior that are not likely to be captured with reductionist models. The goal of this section of the paper is to develop an understanding of true complexity in order to identify particular insights from this field which might help to enrich our approach to engineering modeling and analysis.

Complexity theory attempts to describe and model certain classes of systems, particularly biological systems, which exhibit characteristics such as rich interaction between components, non-equilibrium responses, and emergent behavior. Most authors, when attempting to describe complexity theory, agree that it is difficult to form an explicit definition of a complex system. Instead, general characteristics of complex systems are identified in order to distinguish them 
from merely complicated or difficult to visualize systems. Paul Cilliers identifies 10 such characteristics $^{\mathrm{xix}}$ as shown in Table 1 . While some traditional engineering approaches might capture some of these characteristics (for example feedback loops), many are contradictory to the assumptions typically made in engineering approaches (for example, operating under nonequilibrium conditions).

Table 2. Characteristics of Complex Systems (Cilliers).

\begin{tabular}{|l|}
\hline Complex Systems... \\
\hline ...include large number of elements in system \\
\hline ...include dynamic interactions between elements \\
\hline ...include interactions that are rich (some may be redundant) \\
\hline ...include interactions that are non-linear (small causes can generate large results) \\
\hline ...include interactions that are mostly short range \\
\hline ...include feedback loops \\
\hline ... are open ( interact with their environment) \\
\hline ...operate under conditions that are far from equilibrium \\
\hline ...have a history which is partially responsible for current behavior (evolve) \\
\hline ...include elements which are ignorant of behavior of system as a whole (respond only to \\
local information)
\end{tabular}

Melanie Mitchell notes that these characteristics imply system behaviors that are collective, i.e. they arise from the combined actions of many relatively simple interacting elements without central control. Her definition of a complex system is "a system in which large networks of components with no central control and simple rules of operation give rise to complex collective behavior, sophisticated information processing, and adaptation via learning or evolution." These behaviors are often described as self-organizing. ${ }^{\mathrm{xx}}$ The term "emergent behavior" is also used, since a complex system may exhibit action at the system level that cannot be explained by modeling the individual components. Rather, the behaviors arise from the changing connections between system components. The kinds of systems that serve as examples of this kind of behavior include ant colonies, the human brain, and the national economy.

Most engineered systems are unquestionably complicated, with many interacting parts. But, in practice, most of the interactions in such systems are stable (in the sense that they do not change radically over time) and well-understood. This type of system can be reliably modeled by a reductionist approach. The macro-scale behavior is essentially equal to the sum of the behavior of the parts. A truly complex system, on the other hand, by its very nature cannot be modeled in this way. Its behavior is greater than the sum of the parts and may not be predictable from knowledge of individual component behavior. The configuration of connections determines the response to changing environmental conditions in a dynamic way. Applying a true-complexitybased modeling approach to engineered systems would involve a shift away from the current emphasis on analysis of particular components, towards a better understanding of the connections and interactions between components. 
Complex systems may be distinguished from non-linear chaotic systems, although chaos is sometimes included within the purview of complexity theory. A chaotic system is a non-linear deterministic system in which very small inputs can have large effects (often referred to as the butterfly effect). The behavior of a chaotic system may look random from a certain vantage point, but features of chaotic system behavior have been identified, such as fractal geometry, scalability, and strange attractors, that allow for some analysis and prediction. ${ }^{\mathrm{xxi}}$ Although most engineered systems are not mathematically chaotic, the emphasis on system sensitivity to changes in initial conditions or system parameter values is a helpful perspective from which to conceptualize system models.

Complexity theory, as a relatively recent interdisciplinary scientific enterprise, has up until now remained peripheral to engineering work. Computer engineers tend to be more aware of the field, since it relies heavily on digital computational tools to implement analysis. Expert systems and artificial intelligence comprise one branch of the complex system theory domain (referred to as rule-based models). However the branch of complex system theory that has the potential to enhance our approaches to modeling of engineering system behavior falls into the category of connectionist models. Neural networks ${ }^{\text {xxii }}$ and evolutionary programming ${ }^{\text {xxiii }}$ are two methods that could contribute to engineering analysis, but are not yet widely used for that purpose.

Consideration of complexity theory lends itself to an approach to systems analysis that contrasts with that of reductive modeling. Context has a much more central importance in complexity analysis, since complex systems adapt in response to changes in their environment. Rather than trying to isolate systems from their environment in order to understand them, complex system models must incorporate environmental effects. Complexity theory also emphasizes adaptive or evolutionary approaches to problem solving and distributed, rather than centralized, methods of control.

These concepts are just beginning to filter into domains beyond science where system behavior prediction is important. For example, Swilling and Anneke have recently written a book appropriating a complexity approach for determining ways to respond to the need for sustainability on a global level. ${ }^{\text {xiv }}$ The approach has also been used for analyzing homeland security systems. ${ }^{\mathrm{xxv}}$ Within the engineering education community, complexity theory has been proposed as a unifying approach to understanding and improving the process of engineering education. ${ }^{\text {xxi }}$ Catalano and Baillie have also applied complexity theory to engineering decisionmaking with sustainability as a primary goal. ${ }^{\text {xxvii }}$ The power of complexity theory lies in its nonreductive approach to evaluating and solving problems. The focus is situation-specific, based on narrative, analogy, and metaphor rather than exclusively on global, abstract principles and mathematical models. In this sense, complexity theory applies a post-modern sensibility, rather than the modernist viewpoint that underlies reductive modeling.

\section{Complexity and the Engineering Curriculum}

Engineering students need to be equipped to choose system representations that are fitting for the types of systems they will be designing. For most mechanical systems (no matter how complicated), reductionist models are appropriate. But, the current engineering curriculum relies heavily on deterministic analysis of physical systems as the primary mode of design evaluation. I 
would like to consider what types of modifications to the engineering curriculum would help to better introduce students to the challenges of increasing complexity and help them identify methods for successfully dealing with complexities of all levels. Not all of these suggestions are new, but even those that have been previously proposed by various constituencies within engineering education could use more emphasis and broader application.

\section{Systems Engineering}

Exposing students to the methods and techniques of systems engineering might be a first step in helping them develop skills in decision-making amid complexity. Systems engineering provides techniques for managing complicatedness, encouragement to take a more holistic approach to the experience of problem solving, and particular methods to allow integration of less technical considerations (e.g. usability, serviceability, economics, and management) into the design process. ${ }^{\text {xxiii }}$ It focuses on systems, rather than analysis of isolated elements. However, I am not convinced that systems engineering goes far enough in addressing complexities beyond that of complicatedness. Systems engineering is an extension of the reductionist paradigm, opening the design process toward a more holistic approach, but still based primarily on traditional scientific analysis and optimization tools. Although I would encourage engineering programs to consider adding courses in systems engineering to the curriculum or integrating systems engineering ideas into other technical course (which many programs already have done), as a discipline it has not emerged from a true-complexity theory perspective and therefore may not go far enough in recognizing the limitations of reductionist modeling techniques.

\section{Design Projects}

One way to make students aware of the need to consider alternatives to reductionist models would be to simply provide them more often with comprehensive, realistic, open-ended design problems to solve. The key to the success of this type of exercise in rounding out the modeling toolbox for students would be to ensure that these projects require significant analysis. It has been my experience that often the most open-ended problems assigned are also the ones that involve the least amount of modeling work by students. To emphasize the importance of modeling, students would need to be pushed to develop their designs beyond the conceptual stage. Open-ended projects help students to naturally discover unanticipated interactions by trial and error and would hopefully prod them to appreciate the engineering skills required to address all of the complexities of the design. But, project work of this sort is often frustrating for students, since they must struggle with the challenges of defining system boundaries and selecting modeling approaches without the benefit of knowing the expected outcome. For faculty, it is not easy to determine an appropriate scope for such open-ended projects and the results are difficult to evaluate. Sharing of successful project assignments and grading rubrics among teaching faculty would be helpful in this regard. Design norms should also be used in the context of this kind of problem-solving to emphasize the "people-level" interactions and context which may influence system behavior and therefore need to be better understood.

\section{Complexity Theory Techniques}


If particular techniques of special complexity theory are found to be helpful for engineering system modeling, we will need to determine where to insert them into the curriculum. It may be that only a small number of tools will be relevant and they can be injected into current courses to enhance modeling capabilities. In the same way that writing or ethics is integrated across the curriculum, complex system theory could be integrated into the current content framework. Neural networks, chaotic system behavior, and expert systems, for example, might be topics that could be inserted into the current required courses, although given the packed nature of most engineering programs, this would also entail dropping some other engineering topics. Another approach might be to develop a required or elective course dealing specifically with complexity theory. In the same way that engineering educators are pondering whether new courses in bio-engineering or nano-engineering are needed to meet the changing demands of technological development, a course in complexity for engineers could be considered. Much work will be required to structure such a course and develop supplementary materials that apply specifically to engineering.

\section{A Postmodern Approach to Engineering}

Paul Cilliers, in the last chapter of his book Complexity and Postmodernism, argues that postmodern society itself demonstrates the 10 characteristics of complex systems described above. He directs a warning about the importance of recognizing this condition primarily towards scientists, but I would suggest that this applies to engineers and technology managers as well, especially taking into account the descriptions of technology and complexity provided early in this paper.

"We need to come to grips with complexity in order to ensure our survival. At the same time, complexity is the fount of liberty. In both science and politics, therefore, the acknowledgement of complexity is a vital step. Liberty and justice will not come through the imposition of universal laws by some form of central control, nor will science flourish if it maintains a closed shop and speaks a private language. Scientific activities need to be more open, not only toward the world, but also internally. The barriers between the various scientific disciplines need to be crossed."xxix

Cilliers advocates a connectionist approach toward problem-solving that is situated within a narrative context, rather than abstracted from other aspects of reality. Rather than focusing our attention on drawing tight system boundaries to facilitate reductionist models, a complexity approach should emphasize connections within systems and with the system environment. The knowledge to be gained from modeling and analysis is "determined by participants of the game in terms of their present needs and constraints." $\mathrm{xxx}$ Successful problem-solving involves embracing the dialog between stakeholders and designers.

One traditional modeling technique that could be employed, along with the design norms, to direct this conversation is Failure Mode and Effects Analysis (FMEA). ${ }^{\mathrm{xxi}}$ FMEA works by specifying a structured approach to creatively imagining possible ways a design could fail, as well as the probability and consequences of that failure. FMEA could be an effective method for creatively anticipating previously unanticipated interactions and pointing out needs for alternative modeling strategies to compensate for them. The way FMEA is currently applied, its 
primary advantage is in prioritizing responses to identified failure modes to ensure quality of design (a reductive, check-the-boxes sort of numerical approach), but the method could be redirected to encourage out-of-the-box thinking related to how a particular design might be more complex than originally assumed.

What I am ultimately suggesting is that engineering education, and engineers in general, need to operate from a post-modern paradigm in addition to the traditional approach. Or, perhaps more appropriately according to Bruno Latour, we need to return to a pre-modern sensibility in which the natural connections in the ever expanding collective of non-human things and humans are more explicit, in which "science does no produce itself scientifically, any more than technology produces itself technologically or economy economically."xxxii

\section{Conclusion}

An early reviewer of this paper questioned its relevance to the Liberal Education/Engineering and Society (LEES) Division of ASEE. I would suggest that it is appropriate for this division for two reasons. First, of all the ASEE divisions, LEES is be the most likely to already be familiar with the language of a "postmodern approach" and open to promoting it to students. Second, if a complexity-focused approach to engineering design, one that emphasizes connections and context, is to be developed in students, it cannot be achieved only in technical courses. Both engineering instructors and those in the liberal arts could benefit from consideration of the nature of complexity in technology. This cannot be accomplished without raising the awareness of the existence of complexity theory in the humanities as well as in the sciences. Complexity should be used as a framework for issues raised in humanities classrooms as well as engineering classrooms. We need to exploit the connections between the technical and non-technical aspects of the engineering curriculum.

The dominance of reductionist approaches and methods in engineering education has been built up over the last century or more, and therefore any proposed change to this approach has a great deal of inertia to overcome. Although skill in dealing with complex (in every sense of the word) systems is necessary to engineering practice, the default assumption seems to be that engineering students will have to wait to develop this expertise until they are out on the job. It is hoped that a consideration of the kinds of complexity described in this paper with provide reasons to integrate this practice into student experiences while they are in school.

\footnotetext{
${ }^{\text {i }}$ Shannon Flumerfelt, Gary Halada, and Franz-Josef Kahlen, “Complexity By Design.” Mechanical Engineering, 134 (2012): 28-33.

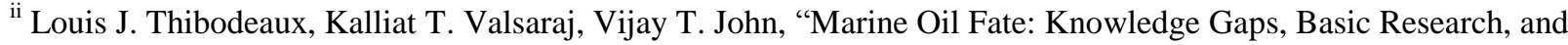
Development Needs; A Perspective Based on the Deepwater Horizon Oil Spill," Environmental Engineering Science 28:2 (2011) 87-93.

iii Oxford Dictionary on-line: http://oxforddictionaries.com/definition/complex?q=complex

${ }^{i v}$ For examples, see "Using Multiple Software Packages To Solve Complex Problems" by Michael Parten, Proceedings of the American Society of Engineering Education (ASEE) Annual Conference, 2003 and "Simulating
} 
Complex Systems In Introductory Dynamics" by Kurt DeGoede, Proceedings of the American Society of Engineering Education (ASEE) Annual Conference, 2005.

${ }^{\mathrm{v}}$ W. Bryan Arthur, The Nature of Technology: What It Is and How It Evolves (New York: Free Press, 2009) Ch. 2.

${ }^{\text {vi }}$ Ibid., 88.

vii Oxford Dictionary on-line: http://oxforddictionaries.com/definition/complex?q=complex

viii For examples, see Nadia Craig, Veronica Addison, Michelle Maher, and Wally Peters, "Integrating Complex Systems Study Into The Freshman Mechanical Engineering Experience," Proceedings of the American Society of Engineering Education (ASEE) Annual Conference, 2005 and Kathleen A. Kramer and Thomas F. Schubert Jr., "Demonstrating Complex Communication Systems Principles Using Electronic Courseware And A Simple Computer Math Package," Proceedings of the American Society of Engineering Education (ASEE) Annual Conference, 1998.

${ }^{i x}$ For examples, see Jennifer Craig, "Teaching Undergraduate Aerospace Engineering Students To Reason And To Communicate About Complex Design Choices," Proceedings of the American Society of Engineering Education (ASEE) Annual Conference, 2006 and Zbigniew Pasek, "It's All There: Teaching Complex Management Content Using Feature Films," Proceedings of the American Society of Engineering Education (ASEE) Annual Conference, $\underline{2008 .}$

${ }^{x}$ Thomas P. Hughes, “Technological Momentum in History: Hydrogenation in Germany 1898-1933,” Past and Present 44 (1969) 132.

${ }^{x i}$ Ingrid H. Soudeck Townsend and Kathryn A. Neeley, “ 'Naming the Complexity': Women's Experience and the Holistic Assessment of Technology," Proceedings of the American Society of Engineering Education (ASEE) Annual Conference, 2000, p. 1.

xii Ibid.

xiii Clive L. Dym, and Patrick Little, Engineering Design: A Project-Based Introduction, $2^{\text {nd }}$ Edition (New York: Wiley, 2004) 24.

xiv Bob Colwell, “Complexity in Design,” IEEE Computer 38:10 (Oct 2005) 10-12.

${ }^{x v}$ George E.P. Box, "Robustness in the strategy of scientific model building," in Robustness in Statistics, R.L. Launer and G.N. Wilkinson, Editors (New York: Academic Press, 1979).

${ }^{\text {xvi }}$ Stephen V. Monsma, ed., Responsible Technology: A Christian Perspective (Grand Rapids, MI: Eerdmans Publishing, 1986) Ch. 9.

${ }_{\text {xvii }}$ The norms in the table have been adapted from the original list in Responsible Technology.

${ }^{x v i i i}$ L. Kalsbeek, Contours of a Christian Philosophy: An Introduction to Herman Dooyeweerd's Thought (Toronto: Wedge Publishing Foundation, 1975) 40-41.

${ }^{x i x}$ Paul Cilliers, Complexity and Postmodernism: Understanding Complex Systems (New York: Routledge, 1998) 3 5.

${ }^{\mathrm{xx}}$ Melanie Mitchell, Complexity: A Guided Tour (New York: Oxford University Press, 2009).

${ }^{x x i}$ Brian Kaye, Chaos and Complexity: Discovering the Surprising Patterns of Science and Technology (New York: VCH Publishers, 1993).

xxii Jet Propulsion Laboratory web-site, "Advanced System Modeling and Control of Bioregenerative Life Support", http://aemc.jpl.nasa.gov/activities/bio regen.cfm (accessed 1/12/2012).

xxiii Terry Bossomaier and David Green, Patterns in the Sand: Computers, Complexity, and Everyday Life (Reading, MA: Helix Books, 1998) 153.

${ }^{x x i v}$ Mark Swilling and E. Anneke, Just Transitions: Exploring Sustainability in a Unfair World (University of Capetown, 2010). 
${ }^{\mathrm{xxv}}$ Alfred A Marcus and Zachary Sheaffer, "Analogical Reasoning and Complexity,” Journal of Homeland Security and Emergency Management 6:1 (2009) article 82.

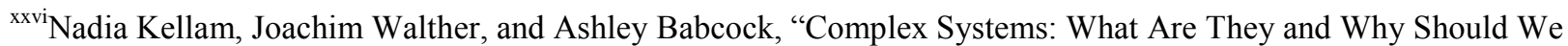
Care?" Proceedings of the American Society of Engineering Education (ASEE) Annual Conference, 2009.

xxvii George Catalano and Caroline Baillie, "Engineering Decisions in the Context of Sustainability: Complex Systems," Proceedings of the American Society of Engineering Education (ASEE) Annual Conference, 2010.

xxviii Benjamin S. Blanchard and Wolter J. Fabrycky, Systems Engineering and Analysis, $3^{\text {rd }}$ Edition (New York: Prentice Hall, 1998) xiii.

xxix Paul Cilliers, Complexity and Postmodernism: Understanding Complex Systems (New York: Routledge, 1998) 127.

${ }^{\mathrm{xxx}}$ Ibid., 129.

${ }^{\text {xxxi }}$ As an introduction to this topic, see The American Society of Quality Engineering web-site: http://asq.org/learnabout-quality/process-analysis-tools/overview/fmea.html.

xxxii Bruno Latour, We Have Never Been Modern (Cambridge, MA: Harvard University Press, 1993) 116. 